\title{
Elastic fields and energies of coherent surface islands
}

\author{
F Jonsdottir ${ }^{1}$, D Halldorsson ${ }^{1}$, G E Beltz ${ }^{2}$ and A E Romanov ${ }^{3}$ \\ ${ }^{1}$ Department of Mechanical and Industrial Engineering, University of Iceland, Reykjavik, Iceland \\ 2 Mechanical Engineering Department, University of California, Santa Barbara, CA 93106, USA \\ 3 A.F. Ioffe Physico-Technical Institute, Russian Academy of Sciences, 194021 St Petersburg, \\ Russia
}

Received 4 September 2005, in final form 11 July 2006

Published 8 September 2006

Online at stacks.iop.org/MSMSE/14/1167

\begin{abstract}
We present simple models based on isotropic elasticity for determining the stress fields in the vicinity of, and the elastic energy associated with, coherent surface islands (i.e. a quantum dot or a nanorod). The first method treats the surface island as an areal point of dilatation and does not account for details of the island shape. Next, the finite element method is used to study simple island shapes such as spherical caps and cylinders, with a particular focus on the island aspect ratio. The latter is used in conjunction with the analytic results to develop empirical expressions for stress field and energy as functions of aspect ratio, which are somewhat insensitive to other features of the geometry. The analyses are used to assess the effect of lattice mismatch, dot volume and dot/surface contact area on the induced stresses and elastic energies. Furthermore, the interaction energy between surface islands is determined by finite element analyses. Outputs from these analyses are then used in an optimization of several cases of ordering of islands. The results show that for a range of idealized geometries, the shape of the surface island has little impact on the strain energy, and thereby the interaction energy.
\end{abstract}

(Some figures in this article are in colour only in the electronic version)

\section{Introduction}

Self-assembled surface nanostructures have attracted considerable attention in recent years (see, for example, the review article by Stangl et al [1]). Examples, including semiconductor quantum dots (QDs) [2], metallic islands [3], nanowires [4], and more recently, nanorods [5], have been intensively investigated. Self-assembled quantum dots (SAQDs) have been of particular interest because of their three-dimensional confinement of charge carriers and excitons and hence their potential in nano-scale electronic devices [6,7]. The fabrication of self-assembled surface nanostructures typically involves crystal lattice mismatch epitaxy of chemically dissimilar materials that results in coherent island formation on the bare substrate surface or on an intermediate thin wetting layer $[2,8]$. Formed at a free surface, coherent islands may then be buried by the growth of the same material as the underlying substrate. Under 
specific conditions, islands can grow upwards, forming nanorods [5,9]. From a mechanical point of view, nanorods, surface islands, and subsurface nano-inclusions act as stressors, that is, sources of elastic strains and mechanical stresses. ${ }^{4}$

Both for understanding mechanical effects on the ordering of surface nanostructures, as well as the effects on electronic properties in SAQD structures, several classes of theoretical and numerical models have appeared and evolved. To name a few, Romanov et al [10] considered the elastic fields of buried islands in subsurface layers. Based on an elastic analysis, it was demonstrated that surface and subsurface stressors can be responsible for the emergence of strain-induced QDs, thus providing, for example, lateral confinement of electrons and holes in quantum wells [11-13]. Subsequent growth of the island material on the buried template has been shown to lead to vertical stacking of islands, provided that the thickness of the intervening layer is on the order of, or thinner, than the lateral dimensions of the island. It has been proposed that dot ordering is driven by the elastic field of the subsurface stressors. Usually, these subsurface stressors are buried dots themselves $[14,15]$; however, they can take the form of subsurface dislocation arrays [16] or buried strained layers on patterned substrates [7,17]. Subsurface stressors lead to a modulation in the stress field and associated strain field on the growth surface, which impacts both adatom diffusion and island nucleation rates $[15,18]$. Ordering inside the layer of SAQDs is essentially governed by the interaction between surface stressors. Shchukin et al [19], with subsequent refinements by MideirosRebeiro and coworkers [20,21], have advanced a model based on statistical thermodynamics that predicts the optimal size and spatial distribution of an ensemble of coherent surface islands.

More sophisticated approaches have been advanced to study self-assembled surface structures. For example, such issues as compositional modulation, separation distances and ordering patterns, and non-uniformity in the size distribution, have been addressed using various techniques. Moreover, there has been an effort to study the effect of anisotropic elastic properties in the island material, including use of the finite element method (FEM), on the mechanical interplay with island behaviour. To illustrate some recent efforts along these lines, Yang [22] and Yang and Tewary [23] used the boundary element method to compute an elastic energy release rate to describe island positions. Yang et al [24] use a local chemicalpotential to describe the self-assembly of QDs. Liu et al $[25,26]$ use a three-dimensional FEM to study the effect of growth rate and elastic anisotropy on the self-assembly of QDs. Furthermore, Zhang [27] and Wise et al [28] demonstrate that surface energy anisotropy may play an important role in the self-organization of QD arrays.

A drawback of the aforementioned studies is that they do not address the behaviour of islands with large aspect ratios, which is of critical importance for nanorods. Before sophisticated models can be explored, a simple approach to understand the mechanical behaviour of self-assembled surface structures is needed. In the present study, we describe the use of small strain elasticity theory to obtain the stress fields of coherent surface islands and the elastic interaction energy between them, with an emphasis on island aspect ratio. Our purpose is two-fold: firstly, by using simple elastic models, we reveal a key geometric scaling of the elastic fields with island shape and size, which suggests that the bulk (elastic) energy of a strained nanocrystal is more complex than a linear scaling with the volume. Secondly, we use the FEM to study the elastic interaction between islands and the effect of the island separation distance on the interaction energy. The results are used to establish an interaction law, that is, energy versus island separation distance. The interaction law is then used in an optimization code to study ordering of multiple islands on the surface of a mismatched substrate. The

4 Even in the case of incoherent interfaces between the island and substrate, elastic fields can develop as a result of thermal expansion mismatch after cooling from the growth temperature. 
study is carried out for several simple, idealized island shapes, such as pyramids, cylinders and spherical caps (all of varying aspect ratios).

\section{Elastic solutions for an isolated surface island}

\subsection{Areal source of infinitesimal lateral dilatation}

A powerful method for the solution of a broad spectrum of problems in elasticity derives from a consideration of point forces applied at some point in an elastic body. On one hand, the concept of point forces can be used as a mathematical abstraction or as a set of elementary solutions (the basis of the so-called Green's function associated with the elastic field equations [29]). With the help of such solutions, one can, for example, find elastic fields of various defect configurations in solids [30,31]. On the other hand, the solutions for point forces can be directly applied to the analysis of a particular physical phenomena, as has been done, for example, in the study of abrupt steps on crystal surface [32], edges of mismatched heteroepitaxial films [33,34], and in the approximate analytical description of the elastic relaxation energy associated with three dimensional strained islands [35].

By applying the point stressor approach to surface islands, we ignore the geometry of the island altogether, and regard it as a point 'spot' of dilatation of strength proportional to crystal lattice mismatch parameter $f$, the strain developed in the island relative to an equivalent parcel of substrate material if it were not constrained by the substrate. That is, if an island with lattice parameter $a_{\mathrm{d}}$ is deposited onto a substrate surface with lattice parameter $a_{\mathrm{s}}$, the mismatch parameter $f$ is taken as $\left(a_{\mathrm{s}}-a_{\mathrm{d}}\right) / a_{\mathrm{d}}$. The effect of the island may be approximated as that of an areal point of expansion, in which two mutually perpendicular pairs of forces (each pair consists of a dipole of opposing forces of magnitude $P$, separated by distance $d$ along their mutual line of action) may be used (see figure 1(a)). The stress field for a single, tangential point force of magnitude $P$ on the surface of a half space is given by Johnson [36]. By superposing solutions for four forces and taking the limit as $d \rightarrow 0$, while maintaining $P d$ constant, one arrives at the following stress field in cylindrical coordinates:

$$
\begin{aligned}
\sigma_{r r} & =\frac{M\left\{2(5+2 v) r^{2} z^{2}-2(2-v) r^{4}-(1-2 v) z^{4}\right\}}{\pi\left(r^{2}+z^{2}\right)^{7 / 2}}, \\
\sigma_{\theta \theta} & =\frac{M(1-2 v)\left(2 r^{2}-z^{2}\right)}{\pi\left(r^{2}+z^{2}\right)^{5 / 2}}, \\
\sigma_{z z} & =\frac{3 M z^{2}(1-2 v)\left(2 z^{2}-3 r^{2}\right)}{\pi\left(r^{2}+z^{2}\right)^{7 / 2}}, \\
\sigma_{r z} & =\frac{3 M r z(1-2 v)\left(3 z^{2}-2 r^{2}\right)}{\pi\left(r^{2}+z^{2}\right)^{7 / 2}}, \\
\sigma_{\theta z} & =\sigma_{r \theta}=0,
\end{aligned}
$$

where $M=P d / 2$ and physically represents the 'strength' of the force quadrapole, $v$ is the Poisson's ratio, $x, y, z$ are Cartesian coordinates introduced in figure 1 , and $r^{2} \equiv x^{2}+y^{2}$. For an actual strained island, the quantities $P$ and $d$ are not straightforward to quantify. Dimensional considerations suggest $M \sim \mu f V$, where $\mu$ is the shear modulus and $V$ is a characteristic volume of the stress field, not necessarily similar to the actual volume of the island.

For the elastic energy associated with a strained island, we first consider the limiting case of an island with very large lateral dimensions compared with its height (so much so that it can be approximated as a thin film). In this case, the island stress field is uniform on the interior of the island, possessing two identical tangential components $\sigma_{x x}, \sigma_{y y}$ of magnitude 


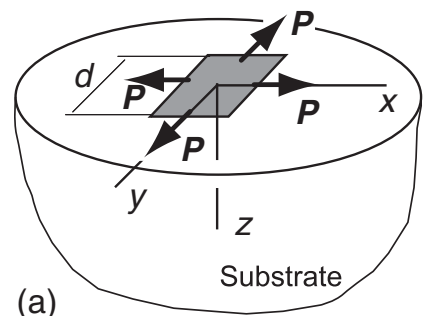

(a)

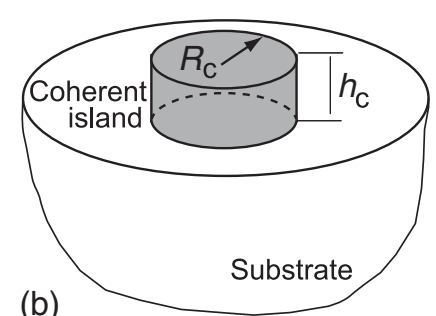

(b)

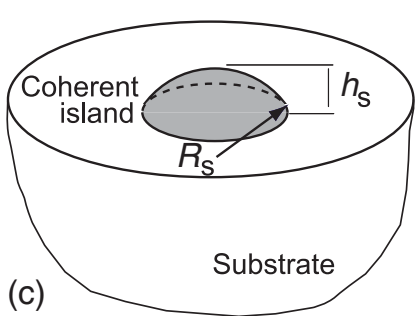

(c)

Figure 1. Simple models for a surface stressor: (a) quadrapole of tangential forces $\boldsymbol{P}$ at the free surface of an elastic half-space; $(b)$ cylindrical coherent island of radius $R_{\mathrm{c}}$ and height $h_{\mathrm{c}} ;(c)$ island with the shape of spherical cap of radius $R_{\mathrm{s}}$ and height $h_{\mathrm{s}}$

$2 \mu f(1+v) /(1-v)$, at least away from the edges of the island. The interior strain energy density is given by $1 / 2 \sigma_{i j} \varepsilon_{i j}\left(\varepsilon_{i j}\right.$ are the strain components given by Hooke's Law; the Einstein summation convention is assumed), and thus the total energy (again, ignoring edge effects) would be given as:

$$
E=2 \mu f^{2} V \frac{1+v}{1-v} .
$$

For more general island aspect ratios, equation (2) could be interpreted as an upper bound on the energy, or alternatively, $V$ could be regarded as an effective volume associated with the stress field in the island and in some part of the substrate adjusting to the island. As implied earlier, this effective volume is not the same as, and generally presumably less than, the actual island volume.

With the aid of a preliminary finite element study and the consideration of data in the literature based on a model two-dimensional system [34,37], we hypothesize that equations (1) and (2) become broadly applicable when $V$ is indeed taken as the effective volume of the stress field in the island and substrate. As will be seen, the volume scales not only with the area $A$ of the island/substrate interface, but on the dimension perpendicular to this interface, physically correlated to the decay of the stress field. The decay length $L_{\mathrm{d}}$ empirically goes as $A^{1 / 2}$ (the characteristic length of the island/substrate interface). Therefore, we have $V \sim A L_{\mathrm{d}} \sim A^{3 / 2}$.

\subsection{Finite element analysis}

To validate our hypothesis on the scaling of stress and energy in a surface island system, we resort to three-dimensional, axisymmetric finite element analysis. The stress fields in the island and the surrounding substrate are evaluated numerically using the commercial finite element software ANSYS [38]. The calculations are carried out for two axisymmetric island geometries: cylindrical islands and spherical caps, shown in figures $1(b)$ and $(c)$. To simulate misfit strain, a condition of continuous displacement is assumed between the island and the substrate. The strain is imparted via a 'phantom' thermal expansion mismatch between the island and the substrate [10]. Of course, in the system we wish to model, dissimilar thermal expansion properties may or may not be significant. However, since the effect of introducing such a differential expansion is to change the zero-stress lattice size for both materials, there is no analytical difference between differential strains induced by lattice mismatch, and those induced by thermal expansion mismatch.

In the interest of brevity, we use the trace of the stress tensor $\left(\sigma_{\mathrm{kk}}\right)$ as a benchmark for comparison. This quantity is important for certain physical phenomena, such as the interaction 


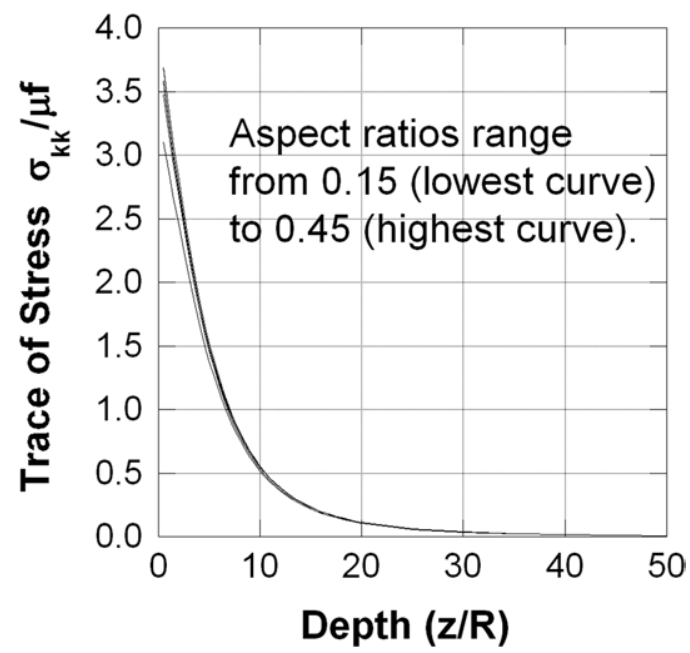

Figure 2. Trace of stress tensor against depth below surface island for various aspect ratios, illustrating the similarity in behaviour across different island shapes, for $v=0.3$. The depth is normalized by $\mathrm{R}$ (either $\mathrm{R}_{\mathrm{s}}$ or $\mathrm{R}_{\mathrm{c}}$, depending on the dot geometry). The dashed curve (not visible) corresponds to the analytical result given by equation (1), with $\mathrm{M}$ is taken as $\beta \mu f A 3 / 2$ and $\beta \approx 0.07$. For aspect ratios greater than the ones shown here, there is no change in the curve.

of point defects with coherent islands. Figure 2 shows the variation of the trace as a function of depth beneath the island, for different values of island height $h$ (and $v=0.3$ ). As expected, the stress asymptotically approaches zero as the depth increases.

Figure 3 shows the normalized strain energy as a function of a dimensionless aspect ratio, $A R=V / A^{3 / 2}$. Note that this aspect ratio increases with an increased island height $h$. The figure shows that the energy reaches a plateau as the aspect ratio increases. Hence, it can be concluded that the energy is relatively independent of the overall volume, except for very shallow islands. It can be argued, on empirical grounds, that equation (1) gives a reasonably good estimate of the stress field beyond several times the characteristic length $\left(A^{1 / 2}\right)$ away from the island, when $M$ is taken as $\beta \mu f A^{3 / 2}$, with $\beta \approx 0.07$; moreover, equation (2) can be simplified as:

$$
E=\alpha \mu f^{2} A^{3 / 2}
$$

where $\alpha \approx 0.20-0.23$. These scalings work well regardless of whether we have considered the spherical caps or the cylindrical geometry for the islands; thus, we conclude that shape is a weak factor in our results. In the cases studied thus far, the coefficients $\alpha$ and $\beta$ are relatively insensitive to geometry and other system parameters. Of course, they have been calculated for one prescribed value of Poisson's ratio $(v=0.3)$. A better theoretical basis for estimating the strength parameter $M$ (and relating it to geometry) is a topic of future work.

\section{Elastic interaction between surface islands}

Interaction energy is the extra energy needed to create an island in a certain location when another island already exists nearby, over and above the energy that is required to create the island when it is isolated. For surface islands of fixed shapes and sizes, the interaction varies only with the distance separating the islands, provided there is no external interference. The essence of the interaction energy is its dependence on the relative distance between the objects. 


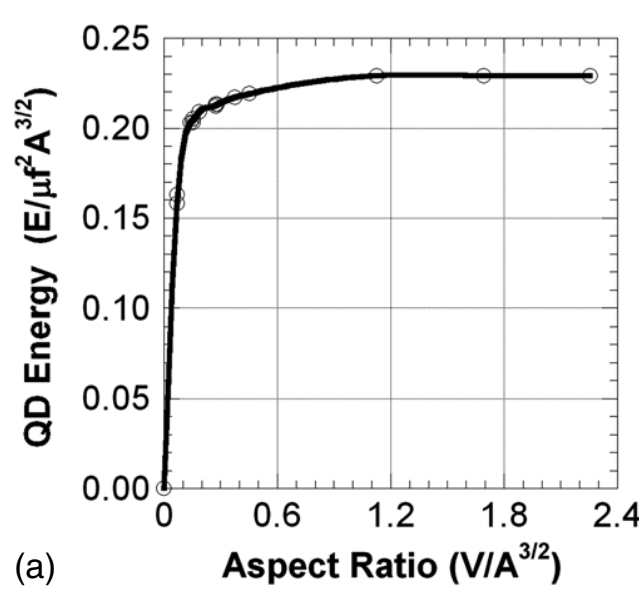

(a)

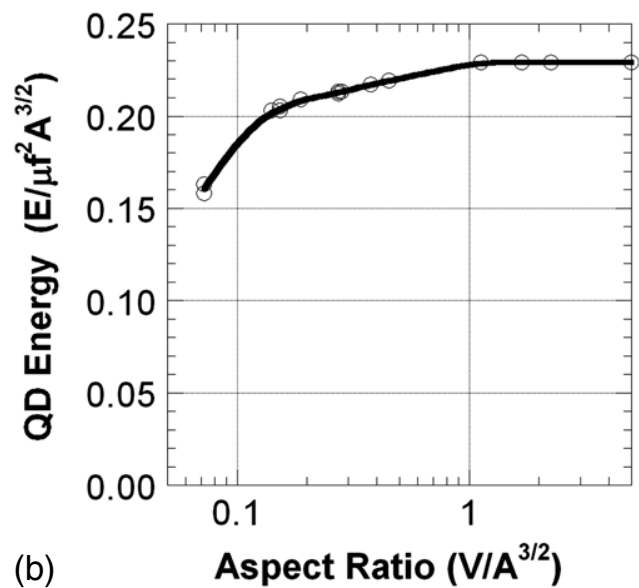

Figure 3. Total, normalized energy as a function of island aspect ratio (one with a log scale on the abscissa is shown to convey more detail in the transition regime). Data points represent a mix of spherical caps and pucks (see figure 1); all fall along the same curve. For aspect ratios larger than approximately 0.25 , the energy (when scaled as such) becomes independent of aspect ratio.

The present study focuses on how interaction energy changes as a function of the distance between the islands for various island shapes and sizes. This will indicate how strongly the islands attract or repel each other. The interaction energy, $I$, is given by:

$$
I=E_{L}-E_{\infty},
$$

where $E_{L}$ is the total strain energy in the substrate and the islands, for a given distance $L$ between the centres of the islands' bases (see figure 4), and $E_{\infty}$ is the total strain energy when $L$ approaches infinity and no interaction exists between the islands.

For now, we consider the simplistic case of two three-dimensional surface islands on an 'infinitely' large substrate. The surface islands studied have the shapes of spherical caps, cylinders and pyramids as shown in figure 4. By taking advantage of symmetry in the problem, only one-half of the object is modelled with the FEM. The element mesh for the islands is kept fine through their entire volume, but for the substrate, the mesh is kept fine in the vicinity of the islands but becomes increasingly coarser in the far field (see figure 4). This is acceptable since the majority of the strain energy is concentrated near the islands. The total strain energy is calculated in the models for various island separations and for various island sizes. The geometry of one of the islands is kept fixed while the other one is altered.

Figure 5 shows the normalized interaction energy as a function of normalized island separation. The results are shown for different island shapes and sizes in a normalized form, where the height of island one is $h_{\mathrm{x} 1}$ and the height of island two is $h_{\mathrm{x} 2}$, where the ' $\mathrm{x}$ ' stands for 'p', in the case of a pyramidical island, 'c' for a cylindrical island and 's' for a spherical island (see figure 4). Similarly, $R_{\mathrm{x} 1}$ and $R_{\mathrm{x} 2}$ give the radii of islands one and two, respectively.

In the cases of cylindrical and pyramidical islands, the $\bigcirc, \times$ and + marked lines represent a change in island/substrate contact area while the lines marked $*, \square, \diamond, \nabla$ represent a change in island height. The spherical cap is in all cases a part of the same sphere, cut at different places to get various ratios of island height versus island radius. As intuitively expected, the interaction energy is high when the islands are close to one another, but it decreases rapidly with increasing island separation and eventually reaches zero when the islands are so far apart that no interaction exists between them. The same trend exists for all shapes and sizes of the islands studied. Figure 5 shows that even though the aspect ratios and types of surface islands 

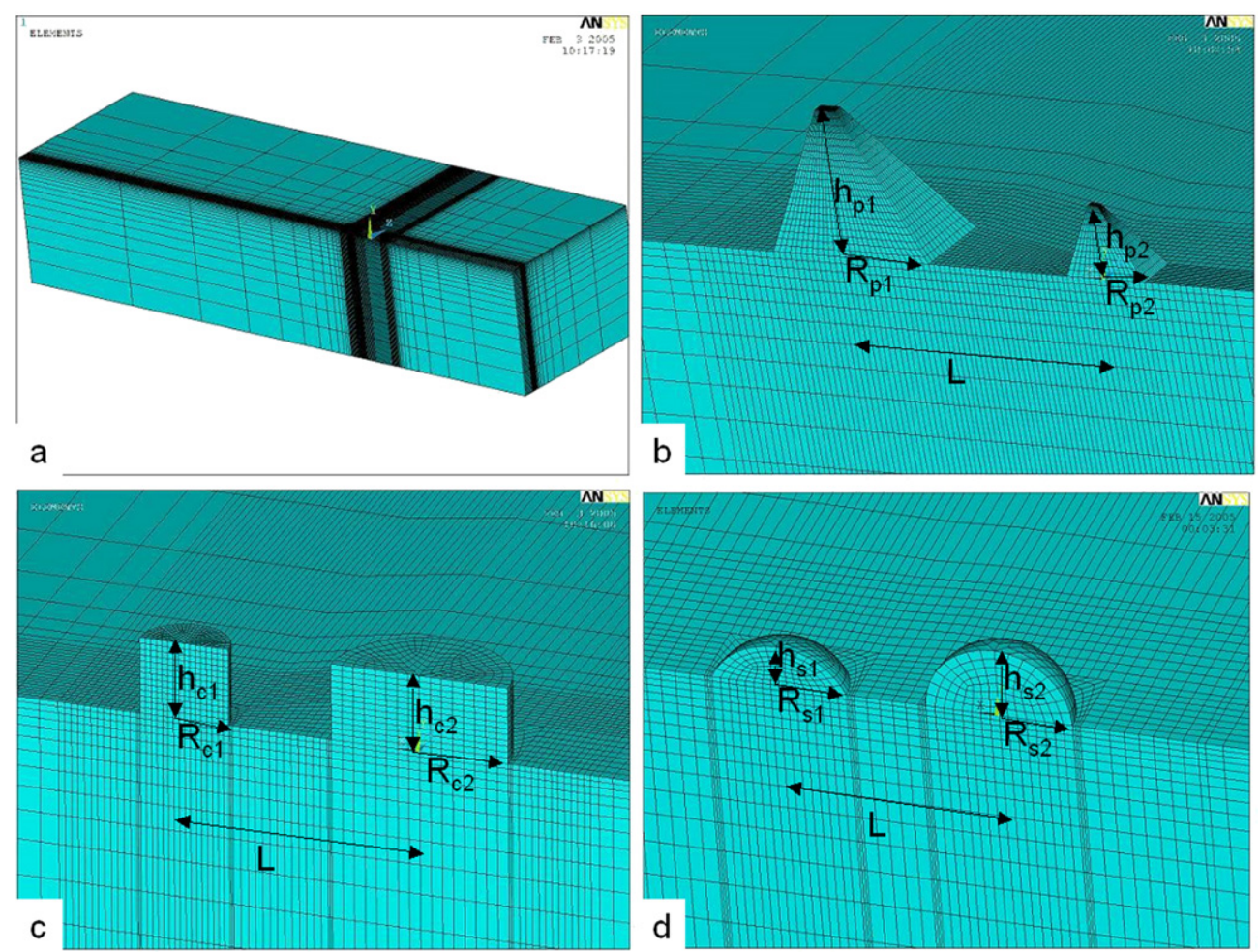

Figure 4. (a) FEM mesh of surface islands on a substrate; (b) pyramidical quantum islands; (c) cylindrical surface islands; $(d)$ spherical surface islands.

are identical, the interaction curves can be quite different. Furthermore, it is evident from the figure that the effect of changing the radii of the cylindrical islands has a much greater impact on the interaction energy than changing the height of the islands. The increase from a flat island to an elongated one produces an increase in the interaction energy, but as the island gets higher, it reaches a certain plateau. This can be intuitively understood by considering how the free surfaces of the islands expand and produce a strain in the proximity of the fixed bases of the islands. The magnitude of that force increases when the island begins to grow upwards from a two-dimensional island, but when a certain plateau is reached, the force (and therefore the interaction energy) remains almost constant.

We now consider the effect of shape of the islands on their interaction energy. Using the aspect ratio $A R=V / A^{3 / 2}$, which was introduced in the previous section, finite element calculations were carried out for pyramidical, spherical and cylindrical surface islands, all of which have the same aspect ratio, $A R=1 / 3$. Figure 6 shows the normalized interaction energy as a function of island separation. The figure indicates that the results, from all island shapes tested, collapse to the same interaction curve. Hence, it can be concluded, at least for the cases tested, that the shape of the surface islands does not have a great impact on the interaction energy.

\section{Ordering of multiple surface islands}

Here, it is assumed that surface islands position themselves on the surface of the substrate, in locations that minimize the total interaction energy. Since analysis with the FEM proved to be 

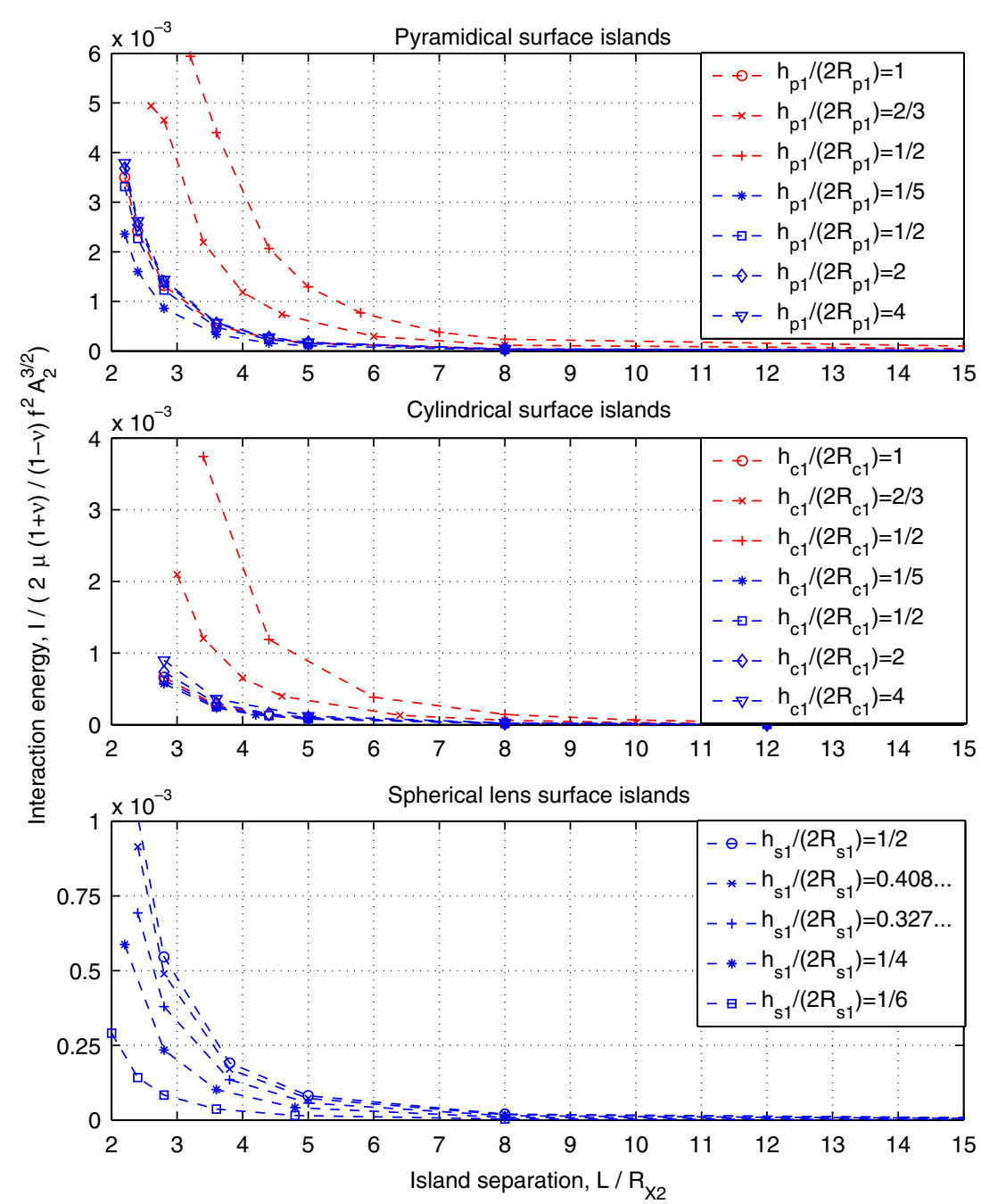

Figure 5. Normalized interaction energy as a function of island separation, for different island shapes and sizes.

time consuming, especially when performing tasks that required multiple iterations, a method was developed to expedite the analysis. The interaction energy was plotted, with results from the FEM, as a function of island separation for all island types of interest, similar to figure 5. Pyramidically shaped islands were chosen for this study. Three types of islands were used with the dimensions $h / 2 R_{\mathrm{p}}=1$ (type 1), 1/2 (type 2) and 1/3 (type 3), where $h$ is the height of the island and kept constant, and $2 R_{\mathrm{p}}$ is the base width (see figure 7 ). Each curve was then fitted with an appropriate equation, which from then was used as a substitute for the FEM to calculate the interaction energy. The total interaction energy for a system of $\mathrm{N}$ surface islands was calculated as:

$$
I_{\mathrm{total}}=\sum_{i=1}^{N} \sum_{j=i+1}^{N} I_{i j}
$$




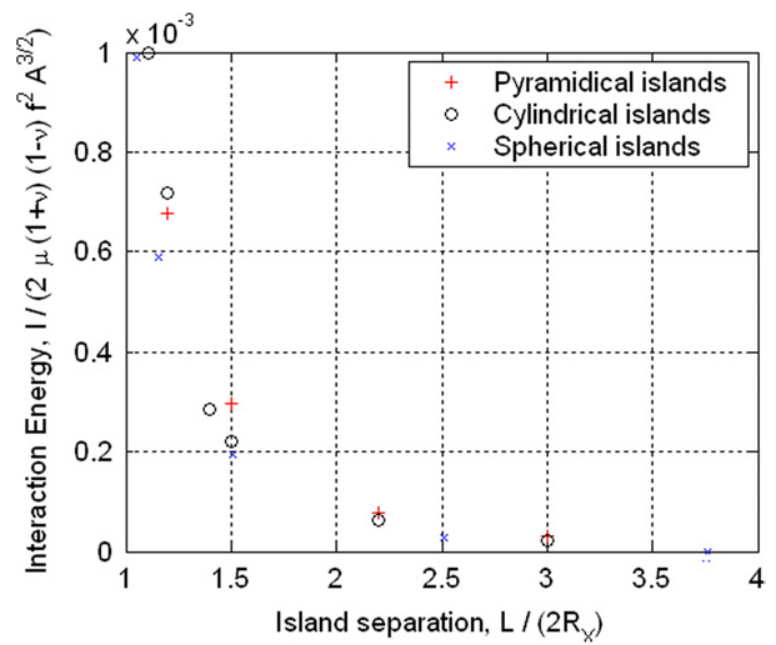

Figure 6. Normalized interaction energy as a function of island separation, for islands with aspect ratio $A R=V / A^{3 / 2}=1 / 3$.

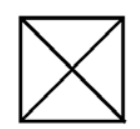

Type 1: $A R=1$

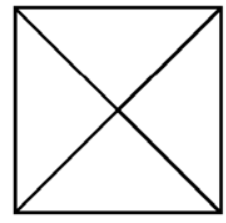

Type 2: $A R=1 / 2$

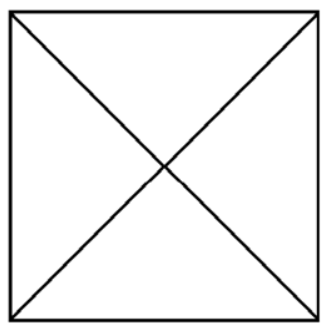

Type 3: $A R=1 / 3$

Figure 7. A top view of the three types of islands used. All are pyramidical with the same height. Islands of type 2 and type 3 have four times larger, and nine times larger base areas and volumes, than type 1 , respectively.

where $I_{i j}$ is the interaction energy between the island $i$ and the island $j$, with due regard for island type in each case. The limits in the double sum have been chosen to ensure that each interaction is counted only once.

A $(1+1)$ evolutionary strategy $[39,40]$ was used to find optimal island positions with respect to interaction energy. When arranging islands on the surface of a uniform substrate that is not previously strained, the optimal arrangement is the one that maximizes the distance between the islands.

\subsection{Positions of stable equilibrium}

The site of stable equilibrium is a position at which the island has no tendency to move to another location in its neighbourhood. We next consider the case of adding a single island to a substrate that already has multiple islands at fixed locations. The island will tend to reposition itself at a location which has a lower interaction energy, that is, it will tend to move to a stable equilibrium position. This position may be one of global minimum in the interaction energy of the system or perhaps at a position coinciding only with a local minimum in the interaction 

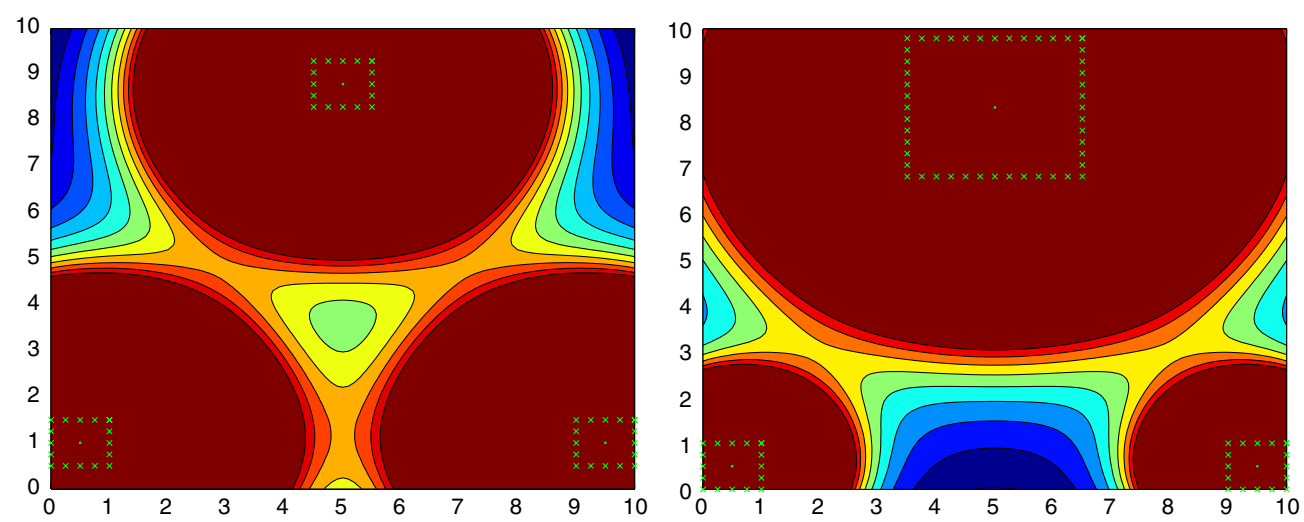

Figure 8. Contour plots of the interaction energy for three pyramidical islands arranged as an equilateral triangle. Two cases are shown: (a) all islands are of type $1 ;(b)$ one island is of type 3 and the other two are of type 1 . The blue (darker) and red (brighter) regions represent regions of lower and higher interaction energy, respectively. The surface islands are shown by green ' $x$ ' marks with islands at their centres.

energy. The equations for the interaction energy, obtained from the FEM, were used to study the interaction energy at all positions between the fixed islands.

First, we consider cases where all islands are of equal sizes and shapes. The cases studied were, for example, three islands fixed at the points of an equilateral triangle, four islands arranged as a square, five islands in the shape of a pentagon and six islands arranged as a hexagon. Figure 8(a) shows a contour plot of interaction energy in the case of three islands arranged as an equilateral triangle. As the figure shows, there is a point of stable equilibrium at the centre of the area. This was true for all cases studied. Hence, it has been shown that there exists a point of stable equilibrium at the centre of the area for an equilateral $n$-polygon when $n=3,4,5$ and 6 . Thus, we hypothesize that this is the case for all $n>2$.

However, this may not be the case if islands are not identical. We next consider an example where the islands are not of equal sizes. Figure 8(b) shows the contour plot of interaction energy for the case of three islands arranged as an equilateral triangle, but now one island is of type 3 and the other two are of type 1. The figure shows that a point of stable equilibrium does not exist at the center of the area as in the previous example.

\subsection{Fixed volume of material dispersed over the surface of a substrate}

An interesting case to consider is the one of how a fixed amount of island material divides itself into either large or small islands to minimize the total interaction energy. Since the interaction energy is much greater for islands with large substrate/island contact area, one might think that the optimal type of islands would be the smallest one, that is, many small islands instead of few large islands. This is, however, not necessarily true because the interaction energy decays with increased island separation (not to mention our omission, in this framework, of the total surface and interfacial energy terms). In the case of many small islands, the island separation is smaller than in the case of fewer larger islands. The number of interactions is given by:

$$
n^{\text {interactions }}=\frac{\left(n^{\text {islands }}-1\right) n^{\text {islands }}}{2}
$$

where $n^{\text {islands }}$ is the number of islands in the system. 

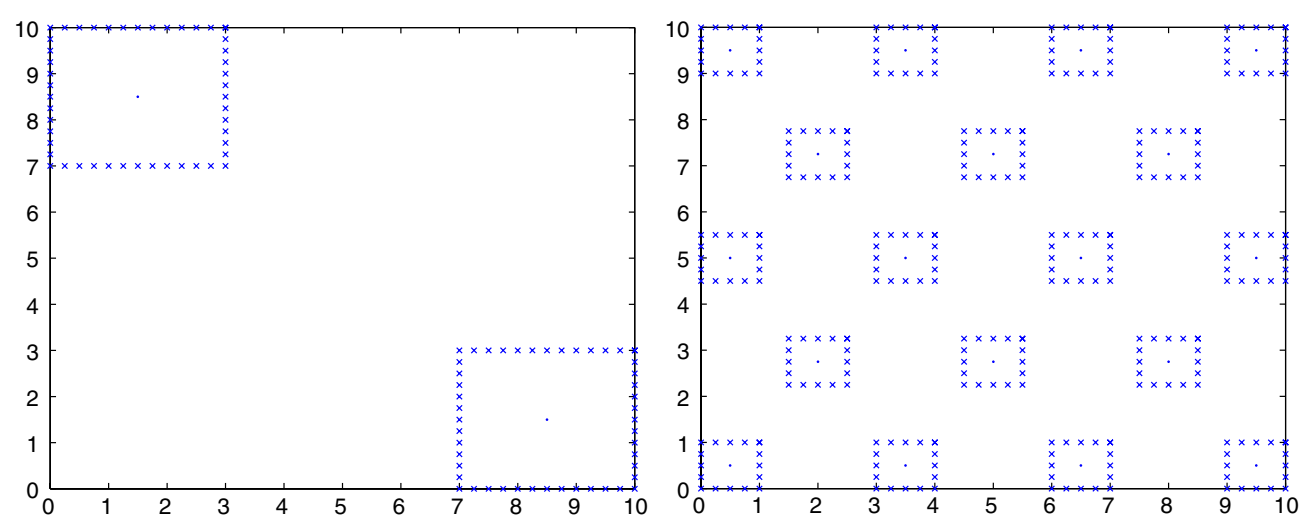

Figure 9. Fixed amount of volume: (a) divided into two large but identical islands; $(b)$ divided into 18 smaller but identical islands.

For now, we consider two possibilities of island formation. Either the island material is divided into two large islands (see figure $9(a)$ ), or into eighteen smaller islands (see figure $9(b)$ ). The islands are all pyramidically shaped with equal height, but the larger islands (figure $9(a)$ ) are of type 3 and the smaller islands (figure $9(b)$ ) are of type 1 .

In this example, the choice is between 18 small islands with 153 interactions (from equation 6) or two large islands. The close spacing of the smaller islands and large number of interactions leads to a higher total interaction energy and therefore the two larger islands represent a favourable configuration. This was true for all cases tested.

\subsection{Mirror boundary conditions}

So far, it has been assumed that no interaction energy exists over the boundaries of the substrate. Consider how the islands are optimized with respect to the mirror boundary conditions for various numbers of islands (see figure 10). The optimization is carried out in such a way that the islands in the original substrate area are iteratively rearranged which leads to repositioning of appropriate islands, because of the mirror conditions. The original substrate is the one in the centre. Although the islands in the original substrate are the only ones that are rearranged, the interaction energy in the optimization is calculated from the total substrate, that is, mirrored islands included.

The islands have the tendency, at least in some parts of the substrate, to arrange themselves in a circular manner over the boundaries. This comes as no surprise since the movement of one of the islands into this circle leads to the movements of other islands into the same circlebecause of the mirror symmetry-which is intuitively energetically not feasible.

\section{Conclusions}

In conclusion, we have shown that equations (1) and (3) offer modestly robust approximations of the stress field and energy of an isolated surface island. Using the FEM to validate the approximations, we found that the results for energy and hydrostatic stress are remarkably independent of island shape for a fixed aspect ratio, at least over the range of idealized geometries considered in this work. A better theoretical basis for estimating the island strength parameter $M$ (prefactor in the expressions for stresses), and relating it to 
(a)

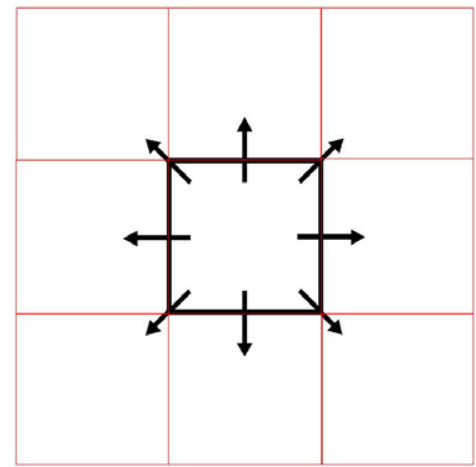

(b)

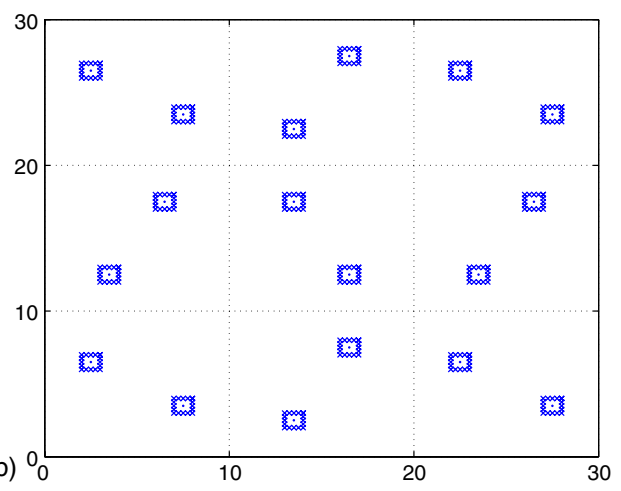

Figure 10. Several islands with mirror boundary conditions: $(a)$ the arrows show the direction of mirror symmetry; $(b)$ two islands with mirror boundary conditions; $(c)$ three islands with mirror boundary conditions; $(d)$ four islands with mirror boundary conditions.

geometry, is a topic of future work. The concepts outlined here have the potential to be used to study the interaction of islands with a broader view towards modelling large assemblages of surface islands. For example, our results can be exploited to study separation distances between islands [23], surface island ordering patterns [3] and non-uniformity in the size distribution. In principle, the simplified approach developed here can be applied to relate elastic effects to compositional profiling in QDs [41]. Most importantly, the results we have obtained serve as an insightful precursor to studies of the interaction of nanorods at various stages during their growth. Various experimental efforts to which our results may be tied include those of $\mathrm{ZnO}$ nanowires on sapphire [5] or $\mathrm{GaN}$ on various substrates, e.g. silicon [9].

Our results additionally show that increasing the island/substrate contact area of an island increases the strain energy in the model. Increasing the height of an island only increases the strain energy up to a certain height. When that plateau has been reached, the strain energy in the model remains constant even though island height is increased. The hydrostatic stress beneath the island decreases with increased depth into the substrate. Discarding size effects, the shape of the island has little impact on the strain and interaction energy in the models. When positions of identical surface islands on the surface of a substrate are optimized, to minimize the total interaction energy in the model, they arrange themselves far apart. In an $n$-polygon with identical surface islands at its corners, there exists a point of stable equilibrium at its center of area. If, however, one or more of the 
islands is larger than the others, this may not be the case. The relatively high interaction energy from the larger islands repels the point of stable equilibrium so the point of lowest interaction energy may lie outside the $n$-polygon. Several cases of dispersion of a fixed volume of island material over the surface of a substrate were studied. In all cases, the more favourable solution was the one with larger islands and thereby less total number of interactions.

The current study can easily be extended to deal with configurations that have not been covered here. Moreover, it could be used to study the effect of anisotropy of the thermal expansion coefficient, buried nano-inclusions and islands grown on wetting layers.

\section{Acknowledgments}

We would like to acknowledge discussions with James S Speck and Krystina Chin. This work was supported in part by the Russian Foundation for Basic Research, Project \# 05-08-65503a (AER) and by the Icelandic Centre for Research \# 040018031.

\section{References}

[1] Stangl J, Holý V and Bauer G 2004 Rev. Mod. Phys. 76 725-84

[2] Leonard D, Krishnamurthy M, Reaves C M, DenBaars S P and Petroff P 1993 Appl. Phys. Lett. 63 3203-5

[3] Brune H, Giovannini M, Bromann K and Kern K 1998 Nature 394 451-3

[4] Gosling T J and Willis J R 1995 J. Appl. Phys. 77 5601-10

[5] Baxter J B and Aydil E S 2005 J. Cryst. Growth 274 407-11

[6] Zunger A 1998 MRS Bull. 23 15-17

[7] Petroff P M 2003 Top. Appl. Phys. 90 1-25

[8] Eaglesham D J and Cerullo M 1990 Phys. Rev. Lett. $641943-6$

[9] Cerutti L, Ristić J, Fernández-Gariido S, Calleja E, Trampert A, Ploog K H, Lazic S and Calleja J M 2006 Appl. Phys. Lett. 88213114

[10] Romanov A E, Beltz G E, Fischer W T, Petroff P M and Speck J S 2001 J. Appl. Phys. 89 4523-31

[11] Tulkki J and Heinamaki A 1995 Phys. Rev. B 52 8239-43

[12] Davies J H 1999 Appl. Phys. Lett. 75 4142-4

[13] Romanov A E, Waltereit P and Speck J S 2005 J. Appl. Phys. 97043708

[14] Xie Q, Madhukar A, Chen P and Kobayashi N P 1995 Phys. Rev. Lett. 75 2542-5

[15] Tersoff J, Teichert C and Lagally M G 1996 Phys. Rev. Lett. 76 1675-8

[16] Romanov A E, Petroff P M and Speck J S 1999 Appl. Phys. Lett. 74 2280-2

[17] Lee H, Johnson J A, Speck J S and Petroff P M 2000 J. Vac. Sci. Technol. B $182193-6$

[18] Mattsson T R and Metiu H 1999 Appl. Phys. Lett. 75 926-8

[19] Shchukin V A, Ledentsov N N, Kopev P S and Bimberg D 1995 Phys. Rev. Lett. 75 2968-71

[20] Medeiros-Ribeiro G 2002 Phys. Stat. Sol. B 230 443-50

[21] Rudd R E, Briggs G A D, Sutton A P, Medeiros-Ribeiro G and Stanley Williams R 2003 Phys. Rev. Lett. 90146101

[22] Yang B 2002 J. Appl. Phys. 92 3704-10

[23] Yang B and Tewary V K 2003 Phys. Rev. B 68035301

[24] Yang B, Liu F and Lagally M G 2004 Phys. Rev. Lett. 92025502

[25] Liu P, Zhang Y W and Lu C 2003 Surf. Sci. 526 375-82

[26] Liu P, Zhang Y W and Lu C 2003 Phys. Rev. B 68035402

[27] Zhang Y W 2000 Phys. Rev. B 61 10388-92

[28] Wise S M, Lowengrub J S, Kim J S, Thornton K, Voorhees P W and Johnson W C 2005 Appl. Phys. Lett. 87133102

[29] Love A E H 1944 A Treatise on the Mathematical Theory of Elasticity (New York, USA: Dover)

[30] Mura T 1987 Micromechanics of Defects in Solids (Dordrecht, The Netherlands: Martinus Nijhoff)

[31] Groves P P and Bacon D 1969 J. Appl. Phys. 404207

[32] Müller P and Saúl A 2004 Surf. Sci. Rep. 54 157-258 
[33] Hu S M 1978 Appl. Phys. Lett. 32 5-7

[34] Kern R and Müller P 1997 Surf. Sci. 392 103-33

[35] Tersoff J and Tromp R M 1993 Phys. Rev. Lett. 70 2782-5

[36] Johnson K L 1985 Contact Mechanics (Cambridge, UK: Cambridge University Press)

[37] Chin K A 2006 Two dimensional finite element analysis of the elastic energy of a quantum island Discovery: UCSB J. Undergrad. Res. submitted

[38] ANSYS v.5.7 (Canonsburg, PA 15317, USA: Ansys Inc)

[39] Rechenberg I 1973 Evolutionsstrategie: Optimierung technischer Systeme nach Prinzipien der biologischen Evolution (Stuttgard, Germany: Frommann-Holzboog)

[40] Schwefel H P 1981 Numerical optimization of computer models (Chichester, UK: Wiley)

[41] Lemaître A, Patriarche G and Glas F 2004 Appl. Phys. Lett. 85 3717-19 